\title{
Orally administered sodium 4-phenylbutyrate suppresses the development of dextran sulfate sodium-induced colitis in mice
}

\author{
KAZUHIKO ONO $^{1}$, SATOSHI NIMURA ${ }^{2}$, YUKO HIDESHIMA ${ }^{1}$, \\ KAZUKI NABESHIMA $^{2}$ and MANABU NAKASHIMA ${ }^{1}$ \\ ${ }^{1}$ Department of Immunological and Molecular Pharmacology, Faculty of Pharmaceutical Science; \\ ${ }^{2}$ Department of Pathology, Faculty of Medicine, Fukuoka University, Fukuoka 814-0180, Japan
}

Received April 13, 2016; Accepted March 23, 2017

DOI: $10.3892 / \mathrm{etm} .2017 .5251$

\begin{abstract}
Sodium 4-phenylbutyrate (PBA) exerts therapeutic effects in a wide range of pathologies. A previous study by the present authors revealed that intraperitoneal administration of PBA suppresses the onset of dextran sulfate sodium (DSS)-induced colitis in mice. In the present study, the effects of orally administered PBA are investigated, as this route of administration is more clinically relevant. The therapeutic efficacy of PBA $(10 \mathrm{mg} / 12 \mathrm{~h})$ in mice with experimental colitis was assessed based on the disease activity index, production of inflammatory cytokines, colon length and histopathological investigations. The results of the present study demonstrated a significantly higher survival rate in the PBA-treated group compared with the PBA-untreated (DSS control) group $(\mathrm{P}=0.0156)$. PBA treatment improved pathological indices of experimental colitis $(\mathrm{P}<0.05)$. Furthermore, the oral administration of PBA significantly inhibited the DSS-induced shortening of the colon $(\mathrm{P}<0.05)$ and overproduction of interleukin (IL)- $1 \beta$ and IL-6 (both $\mathrm{P}<0.05$ ) as measured in colonic lavage fluids. A marked attenuation of the DSS-induced overproduction of tumor necrosis factor was also observed. For histopathological analysis, a marked decrease in mature goblet cells and increase in enlarged nuclei of the absorptive cells was observed in colon lesions of DSS control mice as compared with normal untreated mice. However, in the PBA-treated mice, no such lesions were observed and the mucosa resembled that of DSS-untreated mice. The results of the present study, combined with those results of a previous study, suggest that oral and intraperitoneal administration of PBA have similar preventative effects on DSS-induced colitis, achieved by suppressing its pathogenesis.
\end{abstract}

Correspondence to: Dr Kazuhiko Ono, Department of Immunological and Molecular Pharmacology, Faculty of Pharmaceutical Science, Fukuoka University, 8-19-1 Nanakuma, Jonan-ku, Fukuoka 814-0180, Japan

E-mail:kazu-ono@adm.fukuoka-u.ac.jp

Key words: inflammatory bowel disease, ulcerative colitis, inflammation, sodium 4-phenylbutyrate, oral administration, pro-inflammatory cytokines

\section{Introduction}

Excessive inflammatory responses contribute to and aggravate various autoimmune/chronic diseases, including inflammatory bowel disease (IBD) (1). Crohn's disease and ulcerative colitis are examples of major IBDs of the gastrointestinal tract; both have a similar profile when they present in the colon, including peripheral symptoms such as weight loss and fever, and various colonic specific symptoms including gastric dysmotility, colonic mucosal ulceration, shortening of the colon, and diarrhea (1-4). The imbalance in the mucosal immune response leads to the overproduction of inflammatory cytokines, chemokines, oxidants and matrix metalloproteinases, which in turn results in prolonged inflammatory responses and irreparable tissue damage (5-9). Previous findings have shown the pathogenesis of IBD; however, its etiology remains poorly understood (5-9). Patients with IBD are typically treated using pharmacological agents and strategies, such as anti-inflammatory agents, immunomodulatory therapies, monoclonal antibody therapies and leukapheresis, which target abnormal immune responses and uncontrolled inflammation $(4,10-14)$.

Sodium 4-phenylbutyrate (PBA) is an aromatic fatty acid analog that is typically used to treat urea cycle disorders (15). PBA has also shown potential as a therapeutic treatment in many other diseases, including homozygous $\beta$-thalassemia, spinal muscular atrophy, and a variety of tumors $(16,17)$. It has been reported that PBA may act as a histone deacetylase inhibitor, serve as a chemical chaperone, or act as an ammonia scavenger (17). Furthermore, PBA has been found to suppress endoplasmic reticulum stress and exert anti-inflammatory effects (18-21). The authors of the present study have previously reported that PBA may function as a therapeutic reagent for neurodegenerative disorders, and that intraperitoneal administration of PBA suppresses the onset of experimental murine colitis $(22,23)$.

The aim of the present study was to investigate the effects of orally administered PBA, on colonic inflammation in DSS-induced colitis in mice, which is a standard mouse model of IBD. Oral administration is a more clinically relevant route of administration than intraperitoneal, and so the results of the present study may have practical uses for the treatment of IBD. 


\section{Materials and methods}

Experimental animals. A total of 405 -week-old male ICR mice (28-30 g) were purchased from Kyudo Co., Ltd. (Saga, Japan) and treated as previously published (23). Briefly, the mice were housed in cages (5 mice per cage) at a controlled temperature of $20 \pm 5^{\circ} \mathrm{C}$, relative humidity, $60 \pm 10 \%$ and a 12 -h light-dark cycle. The mice were fed CE-2 (Kyudo) and normal drinking water ad libitum. The animals were divided randomly into the following groups: DSS-non treatment control (normal control; $\mathrm{n}=10$ ), DSS-treated control (DSS control; $\mathrm{n}=10$ ), or DSS-treated with the addition of 5 (PBA 5; n=5), 7.5 (PBA 7.5; $n=5$ ), or 10 mg (PBA10; n=10) PBA every $12 \mathrm{~h}$. Survival, development of DSS-induced colitis, and levels of inflammatory cytokines were analyzed daily during the experiment and colon length and histopathology were evaluated on day 12 . The mice were monitored throughout the experiment every day. All animal experiments were conducted under university guidelines and were approved by the Ethical Committee for Animal Care and Use of Fukuoka University (Fukuoka, Japan).

DSS and PBA treatment. Experimental colitis was induced by the addition of $3.5 \%(\mathrm{w} / \mathrm{v})$ DSS to the drinking water of the mice as previously described (23). PBA (LKT Laboratories, Inc., Saint Paul, MN, USA) was administered orally by gavage at a dose of 5,7.5 or $10 \mathrm{mg}$ every $12 \mathrm{~h}$.

Assessment of DSS-induced colitis. Experimental murine colitis was evaluated using a disease activity index (DAI) as previously described (23-25). Briefly, the DAI assigns a score to weight loss, blood in the stool, and stool consistency. The scoring for weight loss, as the percentage difference from weight on day 0 , was as follows: $0,<1 \% ; 1,1-5 \% ; 2,5-10 \%$; $3,10-20 \%$; and $4,>20 \%$. For stool consistency, scoring was: 0 , normal; 2, loose stool; 4, diarrhea. Finally, scoring for stool blood was as follows: 0 , negative; 2 , Hemoccult positive; 4 , gross bleeding.

Measurement of cytokines using ELISA. Cytokine levels were measured as previously described (23). Briefly, 96-well plates were coated overnight at $4^{\circ} \mathrm{C}$ with the following capture antibodies: Anti-mouse tumor necrosis factor (TNF)- $\alpha$ (cat. no. $14-7325 ; 2.0 \mu \mathrm{g} / \mathrm{ml}$ ), anti-mouse/rat interleukin (IL)-1 $\beta$ (cat. no. 14-7012; $2.0 \mu \mathrm{g} / \mathrm{ml}$ ) and anti-mouse IL-6 (cat. no. 14-7061; $1.0 \mu \mathrm{g} / \mathrm{ml}$; all eBioscience Inc.; Thermo Fisher Scientific, Inc., Waltham, MA, USA). ELISA/ELISPOT diluent as a blocking buffer (250 $\mu 15 \mathrm{X}$ diluent; eBioscience Inc.; Thermo Fisher Scientific, Inc.) was added to each well for $1 \mathrm{~h}$ at room temperature and washed with TBS containing 0.1\% Tween-20 (TBST). The collected colonic lavage fluid using a $1 \mathrm{ml}$ syringe and mouse feeding needle; $100 \mu 1$ of PBS was slowly injected into the rectum, left for $30 \mathrm{sec}$ and collected slowly. The different between individuals was limited and $~ 90 \%$ of the initial PBS was collected. The collected colonic lavage was added at a 1:10 dilution in ELISA/ELISPOT diluent. The plates were further incubated for $1 \mathrm{~h}$ at room temperature and washed with TBST. Detection antibodies $(0.8 \mu \mathrm{g} / \mathrm{ml}$ biotinylated anti-mouse TNF- $\alpha$; 13-7326, $2.0 \mu \mathrm{g} / \mathrm{ml}$ biotinylated anti-mouse/rat IL-1 $\beta$; $13-7112$ and $1.0 \mu \mathrm{g} / \mathrm{ml}$ biotinylated anti-mouse IL-6; 137062; all purchased from eBioscience Inc.; Thermo Fisher Scientific,

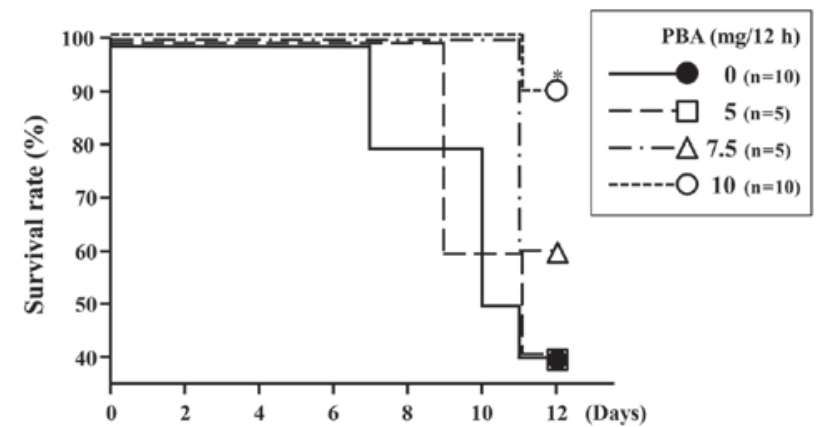

Figure 1. Survival rates of ICR mice receiving 3.5\% DSS with or without PBA for 12 days. ${ }^{*} \mathrm{P}<0.05$ vs. $3.5 \%$ DSS control group. DSS, dextran sulfate sodium; PBA, sodium 4-phenylbutyrate.

Inc.) were added and the plates were incubated for $1 \mathrm{~h}$ at room temperature. Horseradish peroxidase-conjugated streptavidin (SNN 1004; 1:10,000; Biosource; Thermo Fisher Scientific, Inc.) was added and the plates were incubated again for $1 \mathrm{~h}$ at room temperature. Plates were washed with TBST thoroughly, and $75 \mu \mathrm{l}$ of 3,3',5,5'-tetramethylbenzidine (TMB) solution (TMB Microwell Peroxidase Substrate System; SeraCare Life Sciences, Milford, MA, USA) was subsequently added to each well for $10 \mathrm{~min}$ at room temperature, followed by an equal volume of stop solution $\left(1 \mathrm{M} \mathrm{H}_{2} \mathrm{SO}_{4}\right)$. The optical density at $490 \mathrm{~nm}$ was read using an ELISA microplate reader (Bio-Rad Model 450 Microplate Reader; Bio-Rad Laboratories, Inc., Hercules, CA, USA). All anti-cytokine antibodies were purchased from eBioscience Inc.; Thermo Fisher Scientific, Inc.

Measurement of colon length and histopathological evaluation. At the end of the experiment, all the mice were euthanized by cervical dislocation following anesthesia with isoflurane. A section of the colon extending from the cecocolic junction to the anus was harvested as previously described (23). The colon length was defined as the length of the isolated tissue sample as above. The excised tissue was fixed overnight in $10 \%$ neutral-buffered formalin (Nacalai Tesque, Inc., Kyoto, Japan) at room temperature and embedded in paraffin blocks. The paraffin-embedded tissues were cut using a microtome and $5 \mu \mathrm{m}$ sections were stained with hematoxylin and eosin for microscopic examination.

Statistical analysis. Survival data were analyzed using the log-rank test. DAI score data and ELISA data were analyzed by Student's t-test. Colon length data were analyzed by the Tukey-Kramer post-hoc test. Data are presented as the mean \pm standard error of the mean and statistical analysis was performed using GraphPad Prism Software, version 6 (GraphPad Software, Inc., La Jolla, CA, USA). P $<0.05$ was considered to indicate a statistically significant difference.

\section{Results}

Survival rate. At the end of the experiment, the survival rate in the DSS control group was $40 \%$ (4/10). The survival rates of mice treated with DSS and PBA were 40\% (2/5), 60\% (3/5) and $90 \%(9 / 10)$ for the PBA 5, PBA 7.5 and PBA 10 groups, respectively (Fig. 1). The survival rate for mice treated with 
A

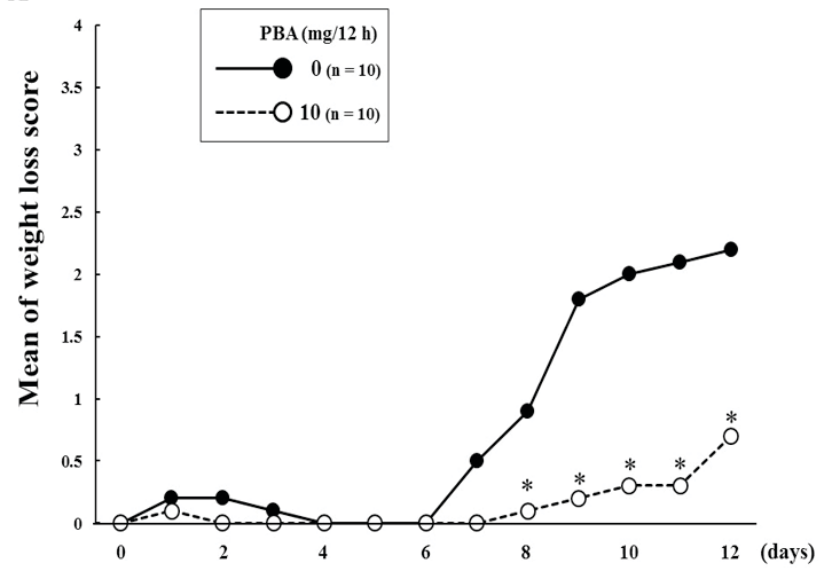

C

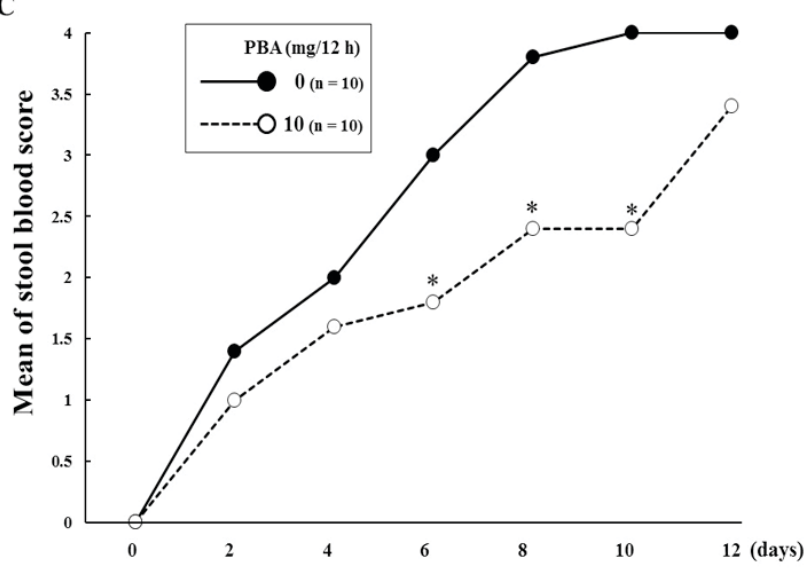

B

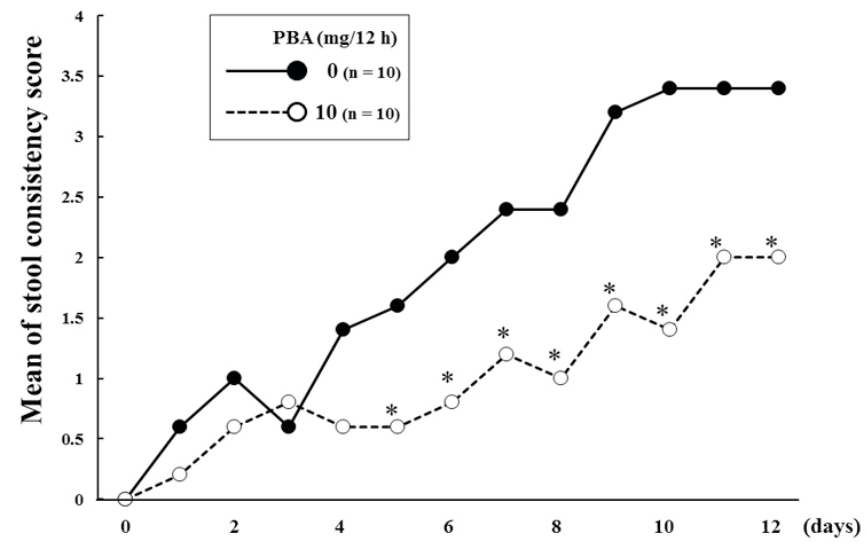

D

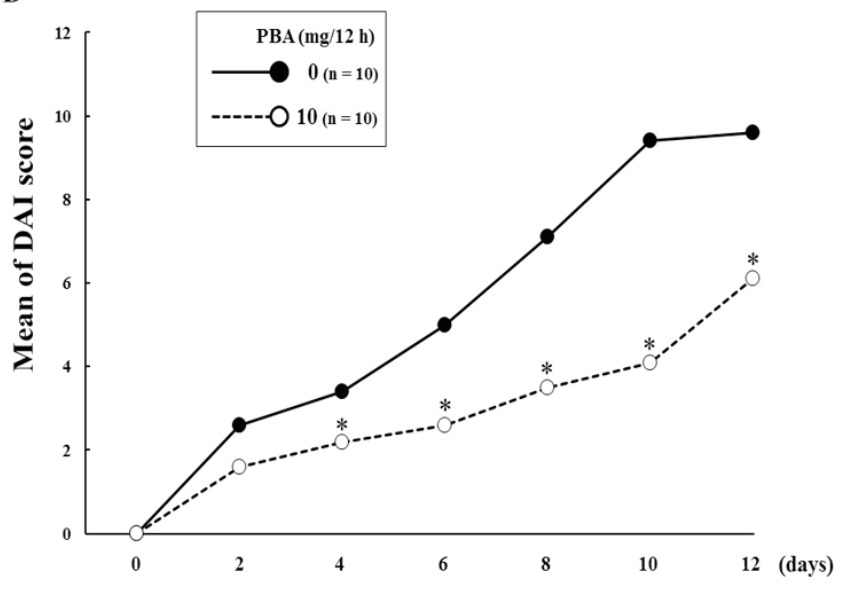

Figure 2. Effects of DSS with or without PBA treatment on the DAI over 12 days. (A) Mean weight loss score, (B) mean stool consistency score and (C) mean bleeding score. (D) Mean overall DAI score. The data are presented as the means \pm standard error of the mean. $n=5$ or 10 per group. ${ }^{*} \mathrm{P}<0.05$ vs. DSS control group. DSS, dextran sulfate sodium; PBA, sodium 4-phenylbutyrate; DAI, disease activity index.

$10 \mathrm{mg}$ PBA per $12 \mathrm{~h}$ was significantly higher than for rats in the DSS control group ( $\mathrm{P}=0.0156$; Fig. 1$)$. In the following experiments, only the results pertaining to the $10 \mathrm{mg}$ PBA per $12 \mathrm{~h}$ group are reported (henceforth denoted as the PBA group), as this was the group yielding the highest survival.

$D A I$. Over the experimental period, there were 5 cases of severe weight loss (DAI score $\geq 3$ ) in the DSS control group. By contrast, there was only 1 case of severe weight loss in the PBA group, which was detected on day 10 (Fig. 2A). The mice in the DSS control group experienced a time-dependent deterioration in the condition of their stools, and ultimately there were 7 cases of diarrhea in the group (score=4). In the PBA group, 9/10 mice had loose stools (score=2) and not diarrhea. One mouse in the PBA group did have diarrhea; however, this mouse did not survive until day 12 (Fig. 2B). In the DSS control group, occult blood (score=2) was observed in $7 / 10$ mice on day 2 and in the remaining 3 mice on day 4. On day 6 , gross bleeding (score $=4$ ) was observed in $5 / 10$ mice. In the PBA group, occult blood was detected in $5 / 10$ mice on day 2 and 8/10 on day 4. On day 8,2/10 mice in the PBA group exhibited gross bleeding (Fig. 2C). Overall, the DAI score of the PBA group was significantly lower than that of the DSS control group from day 4 until the end of the experiment $(\mathrm{P}<0.05$; Fig. 2D).
Inflammatory cytokine production. Cytokine concentration was determined using a standard curve prepared for each plate. In the DSS control group, TNF- $\alpha$ production measured in the colonic lavage fluid increased from day 6 , reaching $335.1 \pm 266.6 \mathrm{pg} / \mathrm{ml}$ by day 8 (Fig. 3A). In the PBA group, TNF- $\alpha$ production was suppressed by $64.2 \%$ on day 8 and $48.6 \%$ on day 10 compared with the DSS control group; however, this was not statistically significant. Similarly, IL-1 $\beta$ production in the PBA group was significantly suppressed on day 10 by $74.5 \%$ (382.4 $\pm 160.6 \mathrm{pg} / \mathrm{ml})$, compared with the DSS control group, in which IL-1 $\beta$ production was $1502.0 \pm 409.2 \mathrm{pg} / \mathrm{ml}(\mathrm{P}<0.05$; Fig. 3B). Furthermore, IL-6 production in the PBA group was significantly suppressed on day 8 by $89.9 \%(167.7 \pm 135.6 \mathrm{pg} / \mathrm{ml})$, compared with the DSS control group, in whichIL-6 production was $1658.7 \pm 600.8 \mathrm{pg} / \mathrm{ml}(\mathrm{P}<0.05$; Fig. 3C).

Colon length. The effect of PBA on DSS-induced shortening of the colon was examined. The length of the colon from the cecocolic junction to the anal verge was measured following sacrifice, and tissues were subsequently processed for histology. The mean colon length was $10.1 \pm 0.3 \mathrm{~cm}$ in healthy control mice, $7.0 \pm 0.2 \mathrm{~cm}$ in the DSS control mice and $8.3 \pm 0.3 \mathrm{~cm}$ in the PBA-treated mice. The difference between the mean colon length in the DSS control and PBA groups indicates a significant suppression of DSS-induced shortening 
A

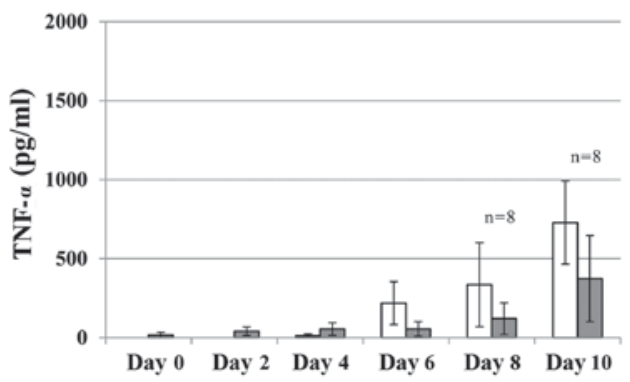

B

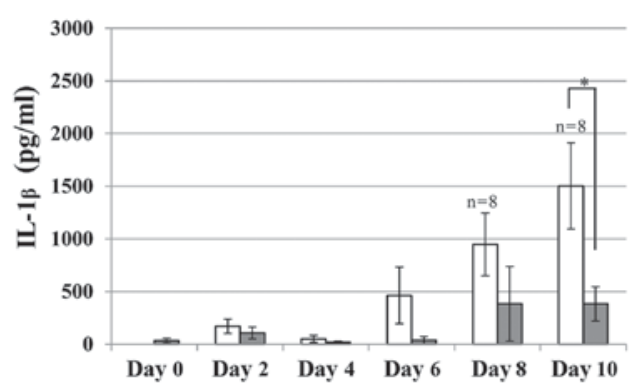

C

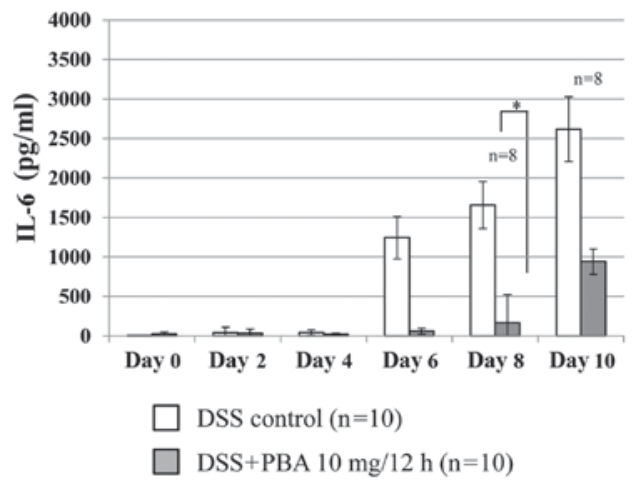

Figure 3. Cytokine production levels in collected colonic lavage fluid. (A) TNF- $\alpha$, (B) IL-1 $\beta$, (C) IL-6. "P<0.05. TNF, tumor necrosis factor; IL, interleukin; DSS, dextran sulfate sodium; PBA, sodium 4-phenylbutyrate.

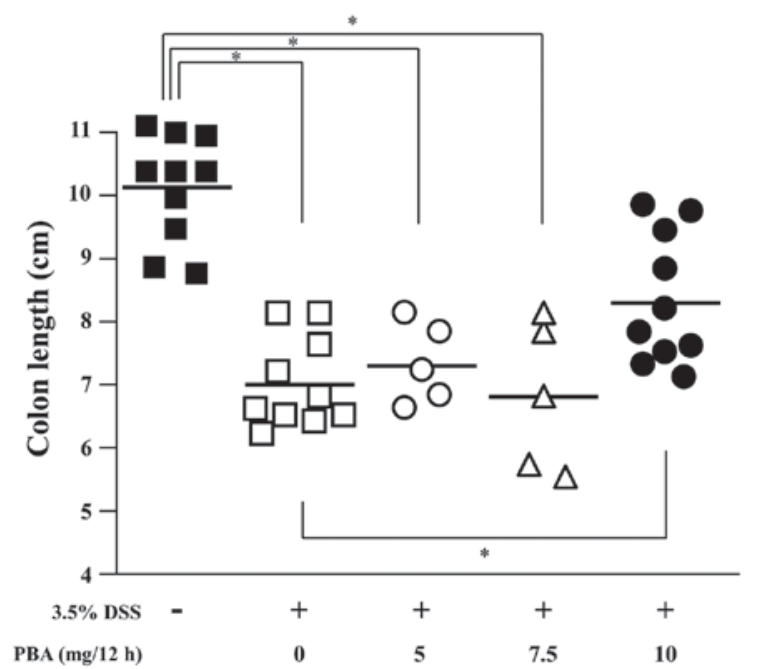

Figure 4. Effect of PBA treatment on colon length. Data are expressed as the mean \pm standard error of the mean. ${ }^{*} \mathrm{P}<0.05$. DSS, dextran sulfate sodium; PBA, sodium 4-phenylbutyrate.

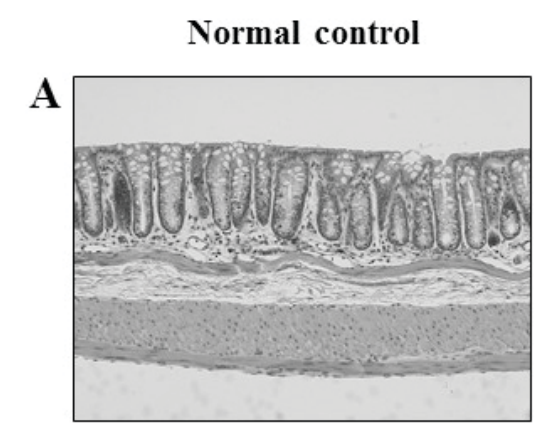

DSS control

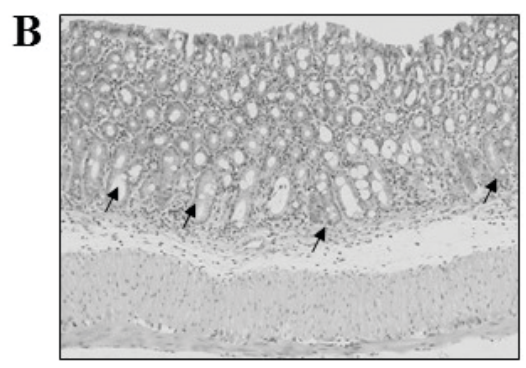

PBA $10 \mathrm{mg} / 12 \mathrm{~h}$

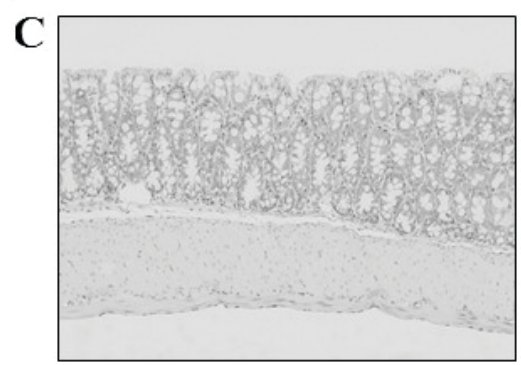

Figure 5. Histopathology of colon tissue. (A) Low-power view of a longitudinal section of a normal colonic wall. Note the crypts with abundant goblet cells. (B) Chronic inflammation in the lamina propria of mice with experimental colitis. Note the loss of goblet cells, the frequently enlarged nuclei of the absorptive cells and the unequivocal mucosal erosion because of the loss of surface epithelia. Loss of goblet cells (indicated by black arrows). (C) No signs of inflammation in the colonic wall of PBA mice. Magnification, x200. DSS, dextran sulfate sodium; PBA, sodium 4-phenylbutyrate.

of the colon in mice treated with $10 \mathrm{mg} P B A / 12 \mathrm{~h}(\mathrm{P}<0.05$; Fig. 4).

Histological findings. Hematoxylin and eosin staining revealed chronic inflammatory infiltrates, including lymphocytes and plasma cells, in the lamina propria around the crypts in the isolated colon segments from the DSS control group. The surface epithelia were partially exfoliated compared with the normal control tissue, revealing the underlying connective tissue of the intestinal mucosa. The crypts contained no mature goblet cells, which indicated mucin depletion (Fig. 5A and B). By contrast, the mucosa from mice in the PBA group had a normal appearance and resembled that of healthy control mice (Fig. 5A and C).

\section{Discussion}

In the present study, the effect of orally administered PBA on experimental colitis in mice was investigated. At the end of 
experiment, the survival rate of untreated mice was low (40\%) whereas, the survival rate of PBA-treated mice was as high as 90\%. Mice in the PBA group had significantly improved scores in the DAI, with PBA treatment markedly inhibiting the decline in weight and deteriorating stool condition. The appearance of a positive hemoccult result or gross blood in the stools manifested more slowly in the PBA group than in the DSS control group. These suggest that PBA treatment delay increased the DAI score and improved the survival rate. PBA also significantly attenuated DSS-induced shortening of the colon and resulted in the maintenance of mucosal integrity to the extent seen in the normal control group. These results suggest that orally administered PBA suppresses or limits the development of experimental colitis in a similar manner to intraperitoneal PBA administration.

In the present study, the concentrations of three important proinflammatory cytokines (TNF- $\alpha$, IL-1 $\beta$ and IL-6) in collected colonic lavage fluids increased in parallel with the worsening of the disease state in the DSS control group. By contrast, PBA treatment markedly reduced TNF- $\alpha$, IL- $1 \beta$ and IL-6 production until day 8. This early inhibition appeared to be sufficient to suppress the onset of experimental colitis, as evidenced by the DAI, histopathological findings and improved survival rates of PBA-treated mice. In the past decade, drugs with anti-cytokine mechanisms of action (including Infliximab) have been applied clinically for the treatment of patients with IBDs (26-28). PBA has the potential to function as an effective therapeutic treatment that is able to inhibit the production of inflammatory cytokines.

The pathogenesis of IBD remains unclear and patients with IBD typically employ symptomatic therapy (5-9). Furthermore, the few medications that are available that relieve symptoms are often associated with inescapable adverse events, which further complicates IBD treatment (4,10-14). In the present study, orally administered PBA was demonstrated to delay the onset of experimental colitis. PBA treatment is not accompanied by the particular side effects of existing IBD therapeutics, and has the added benefit of an oral route of administration (15-21). PBA may therefore be one of a new type of therapeutic agents, which includes anti-TNF- $\alpha$ therapies (29-33). Although the therapeutic effects of PBA appear to involve the suppression of pro-inflammatory cytokines, further studies are required to clarify its precise functional mechanism. The findings of the present study may ultimately allow for the clinical use of orally administered PBA to relieve symptoms and delay the onset of IBD.

\section{Acknowledgements}

The present study was supported by the Central Research Institute of Fukuoka University (grant no. 136003). The authors would like to thank Dr Tomoyo Yasukochi, Dr Takuya Nishinakagawa and Dr Mai Hazekawa for advice on the manuscript.

\section{References}

1. de Souza HS and Fiocchi C: Immunopathogenesis of IBD Current state of the art. Nat Rev Gastroenterol Hepatol 13: 13-27, 2016.

2. Podolsky DK: Inflammatory bowel disease. N Engl J Med 347: 417-429, 2002.
3. Zhang YZ and Li YY: Inflammatory bowel disease: Pathogenesis World J Gastroenterol 20: 91-99, 2014.

4. Leitner GC and Vogelsang H: Pharmacological- and non-pharmacological therapeutic approaches in inflammatory bowel disease in adults. World J Gastrointest Pharmacol Ther 7: 5-20, 2016.

5. Coelho T, Andreoletti G, Ashton JJ, Pengelly RJ, Gao Y, RamaKrishnan A, Batra A, Beattie RM, Williams AP and Ennis S: Immuno-genomic profiling of patients with inflammatory bowel disease: A systematic review of genetic and functional in vivo studies of implicated genes. Inflamm Bowel Dis 20: 1813-1819, 2014.

6. Dupaul-Chicoine J, Dagenais M and Saleh M: Crosstalk between the intestinal microbiota and the innate immune system in intestinal homeostasis and inflammatory bowel disease. Inflamm Bowel Dis 19: 2227-2237, 2013.

7. Ananthakrishnan AN: Environmental risk factors for inflammatory bowel diseases: A review. Dig Dis Sci 60: 290-298, 2015.

8. Maloy KJ and Powrie F: Intestinal homeostasis and its breakdown in inflammatory bowel disease. Nature 474: 298-306, 2011.

9. Scharl M and Rogler G: Inflammatory bowel disease pathogenesis: What is new? Curr Opin Gastroenterol 28: 301-309, 2012.

10. Sands BE: Therapy of inflammatory bowel disease. Gastroenterology 118 (2 Suppl 1): S68-S82, 2000.

11. Ko JK and Auyeung KK: Inflammatory bowel disease: Etiology, pathogenesis and current therapy. Curr Pharm Des 20: 1082-1096, 2014.

12. Pedersen J, Coskun M, Soendergaard C, Salem M and Nielsen OH: Inflammatory pathways of importance for management of inflammatory bowel disease. World J Gastroenterol 20: 64-77, 2014.

13. Katz JA: Management of inflammatory bowel disease in adults. J Dig Dis 8: 65-71, 2007.

14. Yamamoto T, Umegae S and Matsumoto K: Mucosal healing in patients with ulcerative colitis during a course of selective leukocytapheresis therapy: A prospective cohort study. Inflamm Bowel Dis 16: 1905-1911, 2010.

15. Diaz GA, Krivitzky LS, Mokhtarani M, Rhead W, Bartley J, Feigenbaum A, Longo N, Berquist W, Berry SA, Gallagher R, et al: Ammonia control and neurocognitive outcome among urea cycle disorder patients treated with glycerol phenylbutyrate. Hepatology 57: 2171-2179, 2013.

16. Iannitti T and Palmieri B: Clinical and experimental applications of sodium phenylbutyrate. Drugs R D 11:227-249, 2011.

17. Kusaczuk M, Bartoszewicz $M$ and Cechowska-Pasko M: Phenylbutyric Acid: Simple structure-multiple effects. Curr Pharm Des 21: 2147-2166, 2015.

18. Roy A, Ghosh A, Jana A, Liu X, Brahmachari S, Gendelman HE and Pahan K: Sodium phenylbutyrate controls neuroinflammatory and antioxidant activities and protects dopaminergic neurons in mouse models of Parkinson's disease. PLoS One 7: e38113, 2012

19. Luo ZF, Feng B, Mu J, Qi W, Zeng W, Guo YH, Pang Q, Ye ZL, Liu L and Yuan FH: Effects of 4-phenylbutyric acid on the process and development of diabetic nephropathy induced in rats by streptozotocin: Regulation of endoplasmic reticulum stress-oxidative activation. Toxicol Appl Pharmacol 246: 49-57, 2010.

20. Park JS,Lee EJ,Lee JC, Kim WK and Kim HS: Anti-inflammatory effects of short chain fatty acids in IFN-gamma-stimulated RAW 264.7 murine macrophage cells: involvement of NF-kappaB and ERK signaling pathways. Int Immunopharmacol 7: 70-77, 2007.

21. Morinaga M, Kon K, Saito H, Arai K, Kusama H, Uchiyama A, Yamashina S, Ikejima K and Watanabe S: Sodium 4-phenylbutyrate prevents murine dietary steatohepatitis caused by trans-fatty acid plus fructose. J Clin Biochem Nutr 57: 183-191, 2015.

22. Ono K, Ikemoto M, Kawarabayashi T, Ikeda M, Nishinakagawa T, Hosokawa M, Shoji M, Takahashi M and Nakashima M: A chemical chaperone, sodium 4-phenylbutyric acid, attenuates the pathogenic potency in human alpha-synuclein A30P + A53T transgenic mice. Parkinsonism Relat Disord 15: 649-654, 2009.

23. Ono K, Nimura S, Nishinakagawa T, Hideshima Y, Enjyoji M, Nabeshima K and Nakashima M: Sodium 4-phenylbutyrate suppresses the development of dextran sulfate sodium-induced colitis in mice. Exp Ther Med 7: 573-578, 2014.

24. Wirtz S, Neufert C, Weigmann B and Neurath MF: Chemically induced mouse models of intestinal inflammation. Nat Protoc 2: 541-546, 2007. 
25. Cooper HS, Murthy SN, Shah RS and Sedergran DJ: Clinicopathologic study of dextran sulfate sodium experimental murine colitis. Lab Invest 69: 238-249, 1993

26. Dionne S, D'Agata ID, Hiscott J, Vanounou T and Seidman EG: Colonic explant production of IL-1and its receptor antagonist is imbalanced in inflammatory bowel disease (IBD). Clin Exp Immunol 112: 435-442, 1998.

27. Tountas NA, Casini-Raggi V, Yang H, Di Giovine FS, Vecchi M, Kam L, Melani L, Pizarro TT, Rotter JI and Cominelli F: Functional and ethnic association of allele 2 of the interleukin-1 receptor antagonist gene in ulcerative colitis. Gastroenterology 117: 806-813, 1999.

28. Kwon KH, Murakami A, Hayashi $\mathrm{R}$ and Ohigashi $\mathrm{H}$ : Interleukin-1beta targets interleukin-6 in progressing dextran sulfate sodium-induced experimental colitis. Biochem Biophys Res Commun 337: 647-654, 2005.

29. Rutgeerts P, Van Assche G and Vermeire S: Optimizing anti-TNF treatment in inflammatory bowel disease. Gastroenterology 126 1593-1610, 2004.
30. Yun L and Hanauer S: Selecting appropriate anti-TNF agents in inflammatory bowel disease. Expert Rev Gastroenterol Hepatol 3: 235-248, 2009.

31. Bosani M, Ardizzone S and Porro GB: Biologic targeting in the treatment of inflammatory bowel diseases. Biologics 3: 77-97, 2009.

32. Baert F, Noman M, Vermeire S, Van Assche G, D' Haens G, Carbonez A and Rutgeerts P: Influence of immunogenicity on the long-term efficacy of infliximab in Crohn's disease. N Engl J Med 348: 601-608, 2003.

33. Farrell RJ, Alsahli M, Jeen YT, Falchuk KR, Peppercorn MA and Michetti P: Intravenous hydrocortisone premedication reduces antibodies to infliximab in Crohn's disease: A randomized controlled trial. Gastroenterology 124: 917-924, 2003. 